\title{
Brachmann Cornelia De Lange Syndrome: A Tailored Approach for Assessment and Intervention Using the Griffiths III
}

\author{
Jennifer M. Jansen (Corresponding author) \\ Department of Psychology, Nelson Mandela University \\ Port Elizabeth, South Africa \\ E-mail: Jennifer.Jansen@mandela.ac.za
}

\begin{abstract}
Elizabeth M. Green
Association for Research in Infant and Child Development, Manchester, UK

and

Department of Psychology, Nelson Mandela University, South Africa

Louise A. Stroud

Department of Psychology, Nelson Mandela University, South Africa

and
\end{abstract}

Association for Research in Infant and Child Development, Manchester, UK

Mark B. Watson

Department of Psychology, Nelson Mandela University, South Africa

Received: May 12, 2020

Accepted: June 17, 2020

Published: August 1, 2020

doi:10.5296/jse.v10i3.17202

URL: https://doi.org/10.5296/jse.v10i3.17202

\begin{abstract}
The process of evaluation and intervention of an atypical child poses challenges in a number of areas. These challenges include measures that are not normed for a clinical population, the
\end{abstract}




\section{Macrothink}

interpretation of test scores and the use of test scores to devise a meaningful individualized intervention plan that also takes into account sociocultural issues affecting family functioning. To highlight these challenges, an evaluation of a 7-year 1month old girl with Brachmann Cornelia De Lange Syndrome is described using the Griffiths III Scales of Child Development together with the Conners 3- Parent Teacher Surveys and the Vineland Adaptive Scales. The Griffiths III results confirmed a pattern of global delay in all areas of her developmental functioning. The child demonstrated difficulty with the medical and behavioural manifestations of her genetic disorder that needed to be factored into the intervention strategy. The results guided the interventions of different professionals in developing an individualised intervention plan considering the above-identified challenges. The article serves thus as a guide on how to work creatively to determine the level of functioning of an atypical child in the light of the absence of normed measures for such children.

Keywords: Early Childhood, Brachman Cornelia de Lange Syndrome, Griffiths III, Assessment, Intervention. 


\section{Introduction}

Children's normal developmental behaviors follow a predictive course and developmental measures are used to assess these milestones and their delays. The use of these measures aims to intervene at an early stage of a child's development. However, the question arises as to whether children with developmental disabilities can benefit from goal-directed intervention to improve their functioning. Measures that assess childhood development are available but cannot be normed for atypical children unless the normed sample contains only children with a particular condition. A raw score represents the total number of stimulus items that the test taker answers correctly, but such scores are insignificant in interpreting performance as there is no basis for comparison to other test takers' performance. To gain perspective on a child's performance on a norm-referenced test, the examiner must determine how the client performed in relation to other individuals of the same age (Maloney \& Larrivee, 2007). In cases in which age appropriate norms are not available, age equivalents have merit in depicting an individual's range of competencies (Texas Health and Human Services, 2018).

1.2 A developmental assessment using age equivalent scores is a useful guide if a co-morbid manifestation of a child's disability is intellectual impairment. It is important to note that each child, even those who have cognitive delays, have strengths and weakness as part of their clinical picture. Thus, their developmental skills need to be evaluated to plan a way forward for them by instituting an individual support program based on their skill deficits and their relative strengths. This information could then assist in evaluating the additional extracurricular support needed to develop the foundations necessary for learning

1.3 Screening and observational measures are available, but the information gleaned from these measures produces a developmental quotient without any therapeutic information to develop intervention strategies. This raises the concern that we may be testing for the sake of testing and not for intervention purposes. Further, psychologists need to assess how accurate normed measures are in terms of appropriate age when the child being assessed is atypical. Normed measures do not have adaptions such as extra time to complete tasks that would affect the atypical child's performance on the measure. An alternative approach is therefore essential given the challenge when normed measures are used to assist with the education of atypical young children.

1.4 This article will describe the process followed in a case study to develop an individualized support plan with an atypical child diagnosed with Brachmann Cornelia de Lange Syndrome (BCdL). We argue that scores may not be the most important feature of a developmental assessment, but rather the qualitative information gleaned from measures. This information together with collateral data and additional measures should provide sufficient information to develop an initial support plan for an atypical learner. A baseline of developmental functioning can be obtained, which provides an indication of identified deficits to contribute to tailoring an intervention and support plan.

1.5 We argue that scores may not be the most important feature of a developmental assessment, but rather the qualitative information gleaned from measures. This information together with collateral data and additional measures should provide sufficient information to 
develop an initial support plan for an atypical learner. A baseline of developmental functioning can be obtained, which provides an indication of identified deficits to contribute to tailoring an intervention and support plan.

\section{Background to the Assessment}

\subsection{EG's environment}

EG is a 7 year and 1 month old girl living in an impoverished environment in a developing country. She is currently in her first year of formal schooling, not having attended any pre-school education. She thus had not been exposed to an environment, both at home and educationally, that assisted her with the development of the basic skills needed for successful formal learning. Furthermore, the family resides in a crime-ridden area surrounded by extreme poverty. The family is currently functioning in survival mode in all areas of daily living.

\subsection{EG's medical and developmental background}

The background information about EG's developmental history and current functioning was sourced from extensive interviews with both parents, working individually with EG, as well as seeking collateral information from assessment measures. EG was born prematurely at 7 months and hospitalised for an extended period due to her low birth weight. Her mother reported significant delays in reaching developmental milestones. Notably the parents reported that EG did not crawl, but did start walking at the age of two. She spoke her first words at the age of three, and is still not speaking in full sentences. She attained bladder and bowel control after turning four, and managed to stay dry at night at the age of five. The parents also reported consistent breathing difficulties whilst sleeping due to sinus problems. Frequent hospitalisations for both gastrointestinal problems and recurrent fevers occurred, and it was during one of these hospitalisations at the age of four years that she was diagnosed with BCdL. EG's physical appearance and reported history are congruent with the most salient features of BCdL. Her related medical problems have been treated when she had been brought to the emergency room for acute medical emergencies, and she has not been seen on a regular basis at a special clinic at the hospital. During one of these medical consultations, EG's parents mentioned that her teacher had informed them that she was not coping academically in her current mainstream school environment.. Based on the written comments by EG's teacher, her paediatrician requested a psychometric assessment from a community clinic as no psychometric material was available at the hospital.

\subsection{EGs behaviour}

Emotionally the parents reported that EG has extremely labile moods and frequently experienced extreme emotions. They also mentioned that EG seldom sits still and when she does, she resorts to rocking violently and repeating words incessantly. A further worry is that she does not seem able to focus or pay attention on any tasks at home.

2.4 Despite parental collateral history and reported features of possible mental retardation as one of the many features of BCdL, no provision had been made for future academic and other interventions. Although EG is an only child in a supportive family environment, the existing pattern of intervention to date has focused on immediate relief of physical discomfort. Based on this background history, assessment measures were selected to assess EG's behavioral 
manifestations and level of cognitive ability.

\section{Preliminary test battery}

3.1 The Conners 3 - Parent and Teacher Surveys, Long Form was used as both the parents and the teacher reported that EG is highly distractible and shows extremely impulsive behaviour that hinders the learning process. This measure is used to screen for ADHD symptoms and provides information on a range of behavioural and attentional domains. These include inattention, hyperactivity/impulsivity, and indicators of problems with learning, executive functioning, levels of defiance, and peer relations. The test further screens for possible symptoms of Conduct Disorder and Oppositional Defiant Disorder. The scores obtained by both parents and the teacher indicate that EG meets the diagnostic criteria for ADHD Combined Presentation (both inattentive and hyperactive/impulsive) (Conners, 2008).

\subsection{The Vineland Adaptive Behavior Scales 3rd Edition-Survey}

This interview was also included to assess EG's adaptive behavior in the three broad domains measured by the test, namely Communication, Daily Living Skills, and Socialization (Sparrow \& Cicchetti, 1989). It provides an Adaptive Behavior Composite score, which is an indication of overall adaptive functions, and allows for a Maladaptive Index, which screens for undesirable behaviors that may interfere with adaptive functioning. Both parents completed the parent version of this measure. EG's overall Adaptive Behavior Composite score is compiled from the above-mentioned domains, and measures skills essential for everyday living. It also assesses whether these skills can be performed when required. EG's score is representative of a mild deficit and can be described as falling within the lower range. The scores suggest that EG's ability to perform basic everyday skills is poorer than most children her age.

\subsection{Maladaptive Behavior Index}

Due to her academic challenges, reported behavior problems, and general developmental delays, EG was also scored on the that assists in identifying specific behaviors that might be interfering with her ability to perform positive adaptive behaviors. On both the internalizing and externalizing categories EG received scores that were considered clinically significant. She would thus require comprehensive management by her parents, as well as in her schooling environment. The results obtained corroborate her parents' reporting of EG's behavior. It is important to note that this Maladaptive Behavior Index is intended as a screening device, and that EG's behavior warrants further observation and evaluation. It was included in the battery to compare whether the behaviors displayed during the assessment were consistent to those at home.

\section{Method}

\subsection{The Griffiths III Scales of Child Development}

These scales were used to assess EG's current developmental level (Green, Stroud, Bloomfield, Cronje, Foxcroft, Hurter et al. 2016). The Griffiths III provides an overall measure of a child's development, as well as an individual profile of strengths and needs across five subscales, namely (A) Foundations of Learning, (B) Language and 


\section{$\Lambda$ Macrothink}

Communication, (C) Eye and Hand Coordination, (D) Personal-Social-Emotional and (E) Gross Motor domains. While EG's chronological age (7 years 1 month /85 months) technically places her outside the maximum age range of 6 years of the Griffiths III, it was utilized as she presents with atypical patterns of functioning that were expected to fall within the age range of the Griffiths III's developmental information.

The Griffiths III is instrumental in determining whether a child is developing age appropriately or whether general or specific developmental delay is indicated. The Scales provides healthcare practitioners with an opportunity to assist in planning appropriate developmental and therapeutic interventions and shed light on future placement and decisions about the educational management of the child. The Griffiths III is standardized with a normative sample. On a normed developmental measure, scores are of limited use when they fall below the $50^{\text {th }}$ quotient, and clinicians are challenged as to how to report such findings in a manner that will enhance the learning process. This article seeks to assist with interpretation of developmental functioning when the child's scores fall below the $50^{\text {th }}$ quotient and consequently assist with an intervention plan using the developmental constructs not achieved, as well as relative strengths.

4.1.1 Subscale A explores the foundation on which learning takes place by examining the stage a child reached in his or her attempts to comprehend the simplest of objects, qualities in objects, and the relationship between these objects, as well as the applied approach needed in the learning process. This scale evaluates the constructs of Skills of Learning, Ways of Thinking, Memory and Play, all of which form a necessary foundation for cognitive development. The main constructs of the scales are divided into subconstructs within each of the scales. The Skills for Learning construct can be further divided into items that evaluate processing speed, curiosity, flexibility, persistence, creativity, alertness, attentiveness and sustained attention. The Memory construct items evaluate incidental, working, visual and auditory memory, as well as object permanency. The Ways of Thinking construct assesses problem-solving and reasoning and includes items that test the child's reasoning, planning, cause and effect, non-verbal reasoning and concept formation. Play is the last construct assessed on this subscale where the child is encouraged to engage with real objects in an imaginative way.

4.1.2 Subscale B assesses a child's development of speech and language and the ability to use language to engage in social interaction with others. This subscale measures the progression of language and communicative development in all its richness and complexity from pre-linguistic communicative intent to understanding and use of single words, to the development of simple two-word sentences, and then the eventual use and understanding of complex grammar. Language constructs evaluated include receptive and expressive language, verbal reasoning and verbal memory.

4.1.3 Subscale $\mathrm{C}$ is designed to assess a child's visual perceptual skills, fine motor skills and manual dexterity using a range of appropriate tasks. A child's visual motor skills progress from the early development of visual perception, to include an extensive repertoire of different complex movements. This range of skills is developmentally assessed by this measure. Constructs measured in this domain also test visually directed grasp, fine prehension, speed of movement, strength and grip. 


\section{MlMacrothink}

Journal of Studies in Education

ISSN 2162-6952

2020, Vol. 10, No. 3

4.1.4 Subscale D comprises three main constructs that evaluate the Personal, Social and Emotional aspects of the child's development. The Personal construct includes subconstructs pertaining to self-awareness, Theory of Mind, and moral reasoning. The Social construct in this scale measures social interaction, joint attention, humor and play and the Emotional constructs include attachment, moral reasoning and emotional understanding and regulation.

4.1.5 Subscale $\mathrm{E}$ is the gross motor scale and assesses s child's early development of postural control, gross body co-ordination and visual-spatial coordination. In later years, balance rhythm and motor sequencing power and strength are included.

\section{Results}

Considering the overall results on the Griffiths III, EG's scores fall within the extremely low range. EG's consistently low performance across all five subscales indicates significant developmental delays in all areas of development and supports concerns about her academic difficulties. The raw scores she achieved on the test fell below that which is expected of a child aged 72 months. EG's chronological age exceeds the 72 months required of the Griffiths III, making the interpretation of her performance on the measure challenging.

One way of looking at EG's performance in terms of developmental age and relative strengths and weaknesses within each scale is to use the quartile resources developed for the Griffiths III. On these quartile charts, each Griffiths III item has been placed within the year and subscale groupings according to that item's level of difficulty, which was calculated for the standardization sample of typically developing children. The profile of EG's performance on the quartile charts is illustrated in Figure 1.

\section{Griffiths III Year Four Items grouped by Quartile level of difficulty}

ARQDO

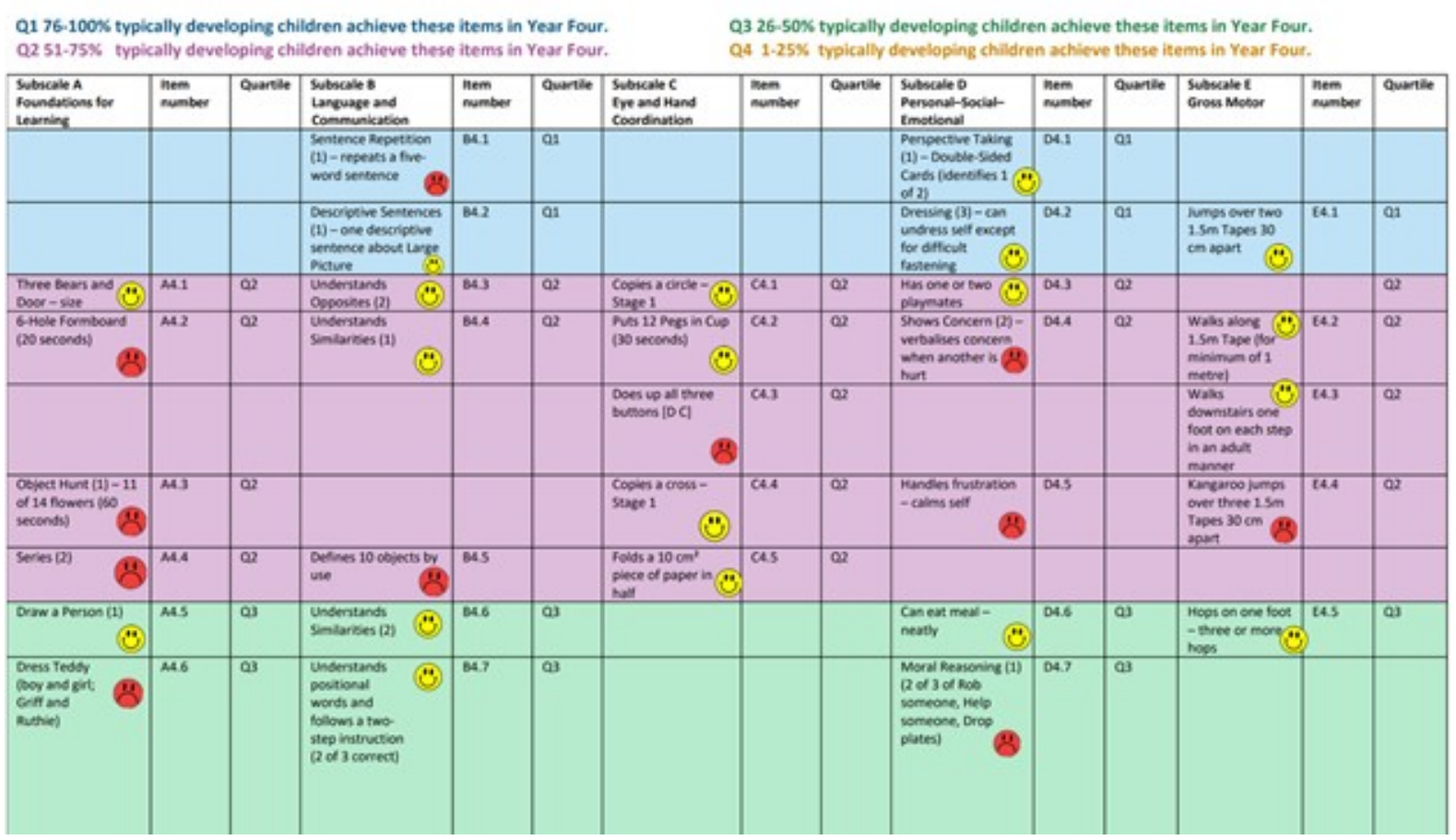

Figure 1. Griffiths III Year Four Items grouped by Quartile Level of Difficulty 
One way to utilize the quartile charts is to examine the developmental age of the items achieved across the five subscales. Another way of using the results is to analyze the constructs not achieved, and to use this information to assist with future educational planning. Figure 1 was used to evaluate the approximate developmental age level EG was able to achieve on the five domains of the measure.

\subsection{Subscale A Foundations of Learning}

When analyzing the results qualitatively, it becomes evident that the constructs that were problematic were 'Ways of Thinking', 'processing speed'and 'concentration'. Her performance on this subscale indicates that EG's executive functioning is compromised. She currently functions at a concrete level with no conceptual thinking present at this stage. Her processing speed was also responsible for errors on timed items. EG found it difficult to persevere with items she found difficult. EG's concentration was better when she was requested to manipulate items in the Quiet Book and other items requiring physical manipulation of her body or of objects.

\subsection{Subscale B Language and Communication}

Her performance indicated that EG's receptive language abilities are better developed than her expressive language skills. When examining the score obtained on the quartile charts, it can be concluded that her expressive language functioning is severely compromised, and her limited speech is possibly affecting many areas of her current functioning, including her labile mood as it is difficult for her to communicate her needs. EG's expressive language was limited to three-word spontaneous utterances, but she was able to understand most of the instructions that indicate some receptive language abilities and she was able to respond to concrete verbs such as "jump", "throw", and other "action-type" verbs. She was able to understand basic similarities and opposites and to identify daily objects and verbs such as running crying, and skipping but not name them.. The general length of her sentences were not longer than three-word utterances. She was not able to repeat a three-word simple sentence. The quartile charts indicate that constructs not achieved include memory concepts such as repetition of a three or five-word sentence, as well as defining objects by use. The charts indicate that $75-100 \%$ of typically developing children aged 45-48 months are able to complete the repetition of a five-word sentence and $51-75 \%$ of typically developing children aged $42-45$ months are able to define objects by use.

\subsection{Subscale C Eye and Hand Co-ordination}

The score suggests that this domain, although poorly developed, is a relative strength when compared to the more intellectual scales. When consulting the quartile charts, EG achieved most of the activities on this scale that are able to be completed by typically developing children up to 48 months, except for fastening three buttons of different sizes. However, when accuracy is necessary to score the item as correct, she is unable to perform these items successfully. It needs to be taken into account that environmental deprivation possibly played a part in the exposure to activities requiring concrete manipulation of objects like dressing dolls and building blocks. EG was introduced to pencil and paper tasks for the first time when she 
attended a formal schooling environment at the age of 6 years. EG is unable to use a pair of scissors and also has problems with bilateral co-ordination.

\subsection{Subscale Personal-Social-Emotional}

EG was able to respond to her name, knew her gender, was toilet trained and could partake in basic self-care tasks. As part of her clinical picture, she presents with an inability to handle frustration and responds to frustration by presenting with autistic symptomology that includes rocking, repetition of the same words and regressed behavior. If she had not been diagnosed with BCdL with autistic like symptoms being a co-morbid feature, further investigations into autism would have been appropriate at this stage. Both EG's teacher and parents confirmed these behaviors and mentioned that the triggers for her behaviors cannot be predicted. However, on a social level, EG has a special friend and she is able to engage in spontaneous parallel play, but she does not understand any type of rules needed for structured interactive play and socialization. The construct that measures D4.4 'Shows Concern' (2) was problematic. The quartile chart indicates that $51-75 \%$ of typical developing children of 48 months or younger pass this item. Emotionally, EG has an inability to recognize and express thoughts and feelings about herself and others, and consequently is unable to benefit from group learning that is interactive in nature. EG was not able to achieve any of the Moral Reasoning constructs on the measure, suggesting a developmental age below the age of 48 months. She was unable to recognize and interpret emotions at any level.

\subsection{Subscale E Gross Motor}

EG was able to perform basic gross motor and visual-spatial coordination activities, such as throwing a tennis ball, and walking up and down stairs and hopping on one foot and was also able to skip without a rope. However, EG presented with midline difficulties such as crossing feet when seated, as well as sequential activities such as hop-jump-hop. Although not age appropriate, this skill is a relative strength for her.

\section{Interpretation of results.}

6.1 As EG's chronological age falls outside of the norm group of this assessment measure, additional methods were used to analyze the constructs achieved or failed, as these constructs are necessary for successful formal learning. The constructs not achieved were analyzed to determine whether a pattern of developmental functioning could be found within the compromised domains. This was necessary in order to assist with the development of an individual support plan.

6.2 Brassard and Boehm (2007) state that score inflation may occur when tests designed for infants and very young children are administered to older preschoolers. Older children with cognitive impairments, who function at earlier developmental levels, may have had a good deal of practice with activities appropriate to those earlier levels. For example, copying a circle may be novel for a 3-year-old, but will not present a mental challenge for a 5-year-old functioning at an earlier developmental level, who may have frequently practised the activity at preschool and thus possibly thus become a learned skill. While there is a possibility that EG has had the necessary stimulation, this is speculative as she spent most of her formative years in an 
impoverished community with parents who have a limited understanding of the value of early childhood development.

6.3 The problem of inadequate test floor (psychometric and clinical) is especially pertinent to the cognitive assessment of young children with intellectual disability (Whitaker, \& Gordon 2018). Many preschool tests do not have norms permitting identification of moderate to profound disability. Even when a developmental score can be obtained, it is sometimes achieved on the basis of only a few credited items if the test has an inadequate clinical floor. Floor effects emerge because the practice of transforming raw scores eliminates any variability present in participants with low intellectual ability (Orsini, Pezzuti \& Hulbert, 2015). This represents a poor sampling of cognitive ability. Instruments providing a sufficient number of developmentally early items, which a child can handle comfortably, will give a better picture of cognitive functioning (Brassard \& Boehm, 2007). As the Griffiths III is developed to assess developmental functioning from birth, there are sufficient items achieved on the measure to provide an indication of EG's developmental level across all five domains.

6.4 The problem of an inadequate test floor (psychometric and clinical) is especially pertinent to the cognitive assessment of young children with a mental disability (Whitaker \& Gordon 2018). Many preschool tests do not have norms permitting identification of moderate to profound cognitive disability. Even when a developmental score is obtained, it is sometimes achieved on the basis of only a few credited items if a test has an inadequate floor. Floor effects emerge because the practice of transforming raw scores into scaled scores eliminates any variability in participants with low intellectual ability (Orsini, Pezzuti \& Hulbert, 2015). This represents a poor sampling of cognitive ability.

6.5 When looking at specific constructs measured in Subscale A, the areas of concern represent the following constructs: sequential and spatial planning, visual and auditory processing speed, visual and memory, arrested learning skills and an inability to work in a mode that requires conceptualisation. When asked to build a train from blocks or a castle using boxes, EG was unable to elevate the manipulation of blocks to build a conceptual object, namely a train. EG also had difficulty when a speed component is necessary to complete an activity, being able to execute the activity, but not within the time limit required.

6.6 Her expressive language was severely compromised and EG essentially presented as a non-verbal child. Scores and constructs not achieved on subscale B suggest that EG's language development needs professional intervention by a speech therapist. An improvement in this area could possible reduce her current emotional regressions, and she may learn to ask for what she needs. It needs to be borne in mind that manifestations of this disorder include severe temper tantrums.

6.7 The Eye and Hand Coordination subscale $\mathrm{C}$ was a relative strength for EG. Her fine motor coordination when manipulating simple concrete objects was adequate. As soon as these activities increased in difficulty, she was unable to complete the higher-order items. Sometimes her poor performance was due to an inability, and other times because of time limits placed on an item in order for it to be scored correctly. EG could reproduce the required shapes with the playdough, place pegs one by one into a beaker, thread beads, but 
she was unable to use plastic strips and bolts to construct a square after a demonstration of how it should be done, or to use a pair of scissors to cut paper. When engaging in pencil and paper tasks, EG was unable to reproduce complex forms like a triangle, draw between two solid lines, or join dots to complete a square or circle. Slow execution speed was evident throughout the assessment. EG's present strengths could assist those working with her to use her learned skills to help her gain greater independence. She needs to be encouraged to fasten her shoes, put on some of her clothes, bath herself, and help with home-based tasks to include her in family activities. A fun element needs to be part of her learning activities.

6.8 On Subscale D, the Personal-Social-Emotional subscale, the scores indicated problems in the recognition of emotions and an inability to show concern when another is hurt. EG had trouble handling frustration and was unable to calm herself. Her poor emotional regulation interfered with all areas of her general functioning. Any type of expectation of EG has the potential to cause extreme distress. She was able to interact with playmates in a free play situation and, while EG has a best friend, she was unable to engage in interactive games requiring rules. EG had difficulty reading cues in social situations.

6.9 Despite EG's performance being compromised on the Gross Motor subscale, the scores obtained were marginally higher than three of the other scales, namely Foundations of Learning, Language and Communication, and the Personal-Social-Emotional Subscale. EG's balance was adequate but not age appropriate. EG executed gross motor tasks like jumping across two tapes and star jumps repeatedly, but she could not perform a sequence of "hop, jump, hop, jump" or coordinate movements where she has to skip with a rope. These deficits can form specialised interventions following both an occupational therapy and physiotherapy assessment.

6.10 The range of these deficits strongly indicate a special schooling environment with fewer children in a class. Her current mainstream environment with a 40:1 pupil ratio is not conducive to learning; in fact this may have exacerbated her present behavioural symptomatology. EG must be taught rules for successful social engagement. She is presently an only child who has not been afforded the opportunity to engage in a preschool teaching environment resulting in limited peer-related interaction. This, together with the dangerous environment where she resides and her cognitive limitations, make her vulnerable in social situations. Small group interactions in a learning environment will provide a safe space to experiment with expectations, rules, and consequences of unacceptable behaviours, as well as time to learn and complete tasks.

\section{Case conceptualization}

The assessment results suggest interventions in three areas of EG's general functioning, namely cognitive, behavioral and medical domains. It is evident that EG is unable to continue schooling in a mainstream environment and needs special school placement. The special school must be able to provide multidisciplinary interventions to address the domain deficits in her functioning. The team that would be most beneficial should include a special needs teacher, an occupational therapist, a nurse, a psychologist and possible a physical therapist. The recommendation of special schooling is based on literature that suggests that most 


\section{Macrothink}

affected individuals have a mild-to-moderate intellectual quotient of 30-85, with an average of 53 (Swols, Tekin, Macdonald, \& Hussman, 2019). Broad goals when considering educational planning of such children are to take into account their stamina, their ability to manage sensory-motor demands, their communicative status, and the need for structure and organization (Cornelia de Lange Foundation, 2018). Goals and objectives of the child's education plan must be individualized in order to keep them motivated. Many of the activities need adaption to meet the needs of these children. Moreover, the child with BCdL needs to be an equal partner in interactive and satisfying social experiences (Cornelia de Lange Foundation, 2018.)

Figure 2 states the manifestations of EG's current profile and highlights interventions necessary in all these domains. Most of the medical interventions will take place outside the school environment facilitated by a nurse who is a member of the health care professions functioning in the special school. The developmental interventions include therapeutic and teaching interventions based on the information obtained. The behavioral aspects that impede learning will be handled by all staff dealing with EG under the guidance of the psychologist. Although interventions seem siloed, consistency, communications and reinforcement form an integral part of her successful support plan and must be reinforced by all members of the support team. The last section of the figure describes multiple socio-environmental challenges that are barriers to EG receiving the education needed. The focus of the discussion in this article is on school-related intervention, whilst also describing other factors that need intervention, followed by a brief discussion regarding challenges. 


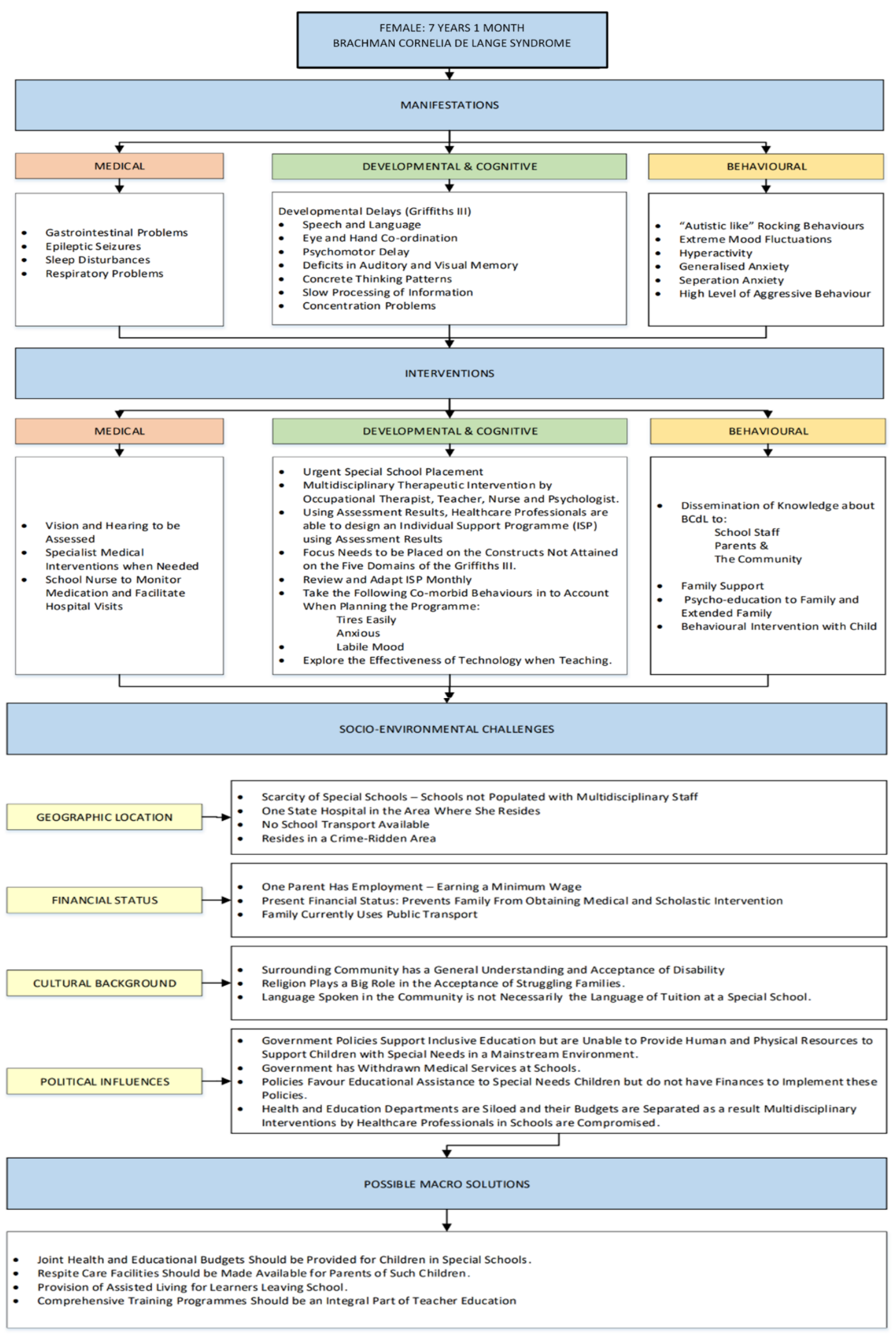

Figure 2. Case Conceptualization 


\section{Interpretation of results}

\subsection{Medical Interventions}

The medical manifestations of BCdL need consistent clinical attention throughout EG's life; including a geneticist, cardiologist, gastroenterologist, nutritionist, ophthalmologist, hearing specialist and neurologist. At present, EG's immediate medical needs are gastrointestinal in nature, sporadic respiratory problems, chronic low energy and infrequent epileptic seizures. At the time of the developmental assessment, her parents and present teacher did not highlight EG's hearing and sight as problematic, possibly because she had not attended a pre-school which would have provided an opportunity for this type of observation. Assessment of these modalities should be attended to as a matter of urgency, as deficits in these areas could affect learning.

As BCdL is a chronic condition, the medical anomalies that occur need to be addressed by medical healthcare professionals primarily in a medical setting. There is however a dire need for family, medical-health personnel and school staff to communicate with each other around issues affecting health. School staff need to be sensitive to those periods when there is a flare-up of gastro-esophageal reflux. Staff also should be informed of food allergies and strictly adhere to dietary limitations. Lastly, staff needs to distinguish between those periods of fidgeting and non-attentiveness due to discomfort and pain versus those periods when these behaviors are due to other causes.

\subsection{Developmental and Cognitive Interventions}

This section addresses the crossover aspects of interventions of this team, as well as the information that has proved useful when working with children diagnosed with this disorder. Interventions involving EG's developmental and cognitive domains need attention as her overall developmental functioning falls below 4 years 7 months, and her overall scores fall below the $50^{\text {th }}$ quotient. It was mentioned earlier that EG's age falls outside of the age range for which the Griffith III has been normed, and the measure has not yet been normed for clinical population groups. However, both the quartile charts and the developmental constructs assessed are able to give an indication of the age level of functioning on the five domains discussed in the article. The Griffiths III has an added advantage of attaching a learning construct to each item assessed, thus enabling an analysis of items at each age in relation to reach of the five domains that were not achieved. This measure, together with clinical observations and other supplementary measures during the evaluation, can assist with the development of an individual support plan for her. EG's developmental profile is globally delayed.

8.2.1 The most academic domain, that of Foundations of Learning, highlighted the concrete nature of EG's thought processes and her inability to think both flexibly and conceptually. Many basic concepts not attained on the Foundations of Learning subtest need to be taught by the class teacher in a specialised learning environment. EG's classroom situation should resemble a preschool classroom where the skills required for learning need to be consolidated in a play environment. Exposure to listening exercises, activities requiring hand-eye 
co-ordination, interactive activities involving turn taking and sharing, should all form part of her day. Introduction to fantasy play and spontaneous outside play are important for her development. Craft and drawing activities should be introduced to improve fine motor skills. These activities should include concrete and pencil and paper tasks. The activities should revolve around the recommendations of the occupational therapist to promote consistency. Should a physiotherapist be part of the multidisciplinary team, inputs from this professional should be built into her daily programme. Language needs constant reinforcement during all activities. The teacher has the most difficult task; he/she needs to provide opportunities to reinforce concepts addressed by both the occupational and speech therapists in the classroom, as well as teach constructs not achieved on the Foundations of Learning subscale, as these form the foundation for successful learning. The teacher must also manage EG's co-morbid behavioural manifestations of hyperactivity, and her difficulty with emotional regulation. For EG's individual support plan to be relevant, multidisciplinary meetings between the therapists involved in her education and the teacher must occur on a regular basis. Challenges and adaptations to EG's individualised teaching plan have to be regularly discussed and amended, based on information gathered from all health care professionals working with her.

8.2.2 The area of development needing intensive intervention is that of speech, receptive and expressive language, as well as encouragement to participate with other children using language if this is possible. The focus of intervention by the speech and language therapist can be guided by the constructs not achieved in this developmental domain. The therapist will also need to evaluate whether EG will acquire adequate speech to interact and learn, and if assistive language devices such as a word board or technology could assist with the process of learning language. EG's scores on the Speech and Language subscale indicate severely specific deficits in expressive language. Severe speech delay or absent speech is typical ofBCdL. Approximately one half of children with BCdL aged 4 years or older combine two or more words into sentences, one third have no words or only 1-2 words, and only $4 \%$ have normal or low-normal language skills (Swols et al., 2019). Children with BCdL have difficulty in understanding the subtle nuances and pragmatics of language and auditory sensitivity to a barrage of speech sounds. Speech is difficult for many of these children due to oral-motor apraxia. These children choose not to talk as they find the coordination and production of the motor actions difficult, especially under typical school demand situations.

8.2.3 Medical interventions in BCdL are usually the initial interventions that spontaneously occur because of growth delay in utero, a strong possibility of premature birth, and feeding difficulties. This aspect has given rise to medical literature being dominant in this area of intervention, while other healthcare and teaching professionals have provided limited academic literature as to how to address therapeutic and scholastic deficits in other areas of functioning.

8.2.4 Many of these children also have hearing impairment as well as abnormal speech development. Middle ear infections sometimes occur chronically. An interesting phenomenon related in a retrospective study by Janek et al. (2016) indicated that hearing loss in BCdL syndrome can improve over time. More than half of the study's 78 patients, seen in an adult $\mathrm{BCdL}$ syndrome clinic, reported hearing improvement, including a subset of patients with 
sensorineural hearing loss. As there are no results available concerning younger children with this disorder, regular audiology consultations may be necessary. Computer programs that emphasize visual memory are more beneficial than standard methods of verbal instruction, and perceptual organizational tasks should be emphasized (Swols et al. 2019). Tactile stimulation during indirection helps children remember and perform maximally. Cameron and Kelly (1988) report a case of a 2-year, 7-month-old girl with BCdL, normal intelligence, and age-appropriate language skills. She demonstrated initial delays in gross motor skills and in receptive and expressive language but responded well to intensive speech and language intervention. This favorable outcome provides an important example of the beneficial effects of therapy, even for clients with conditions generally believed to have a poor prognosis. Thus, evidence-based research suggests that it would be useful for EG's hearing to be check regularly (Cameron \& Kelly, 1988).

8.2.5 Speech may not be a realistic goal for some children. However, other educational techniques can be employed for effective communication and are reliable regardless of the country or culture. Signing may not be an effective route due to receptive difficulties such as poor visual attention, a visual impairment, and lor the transient, spatial or speed nature of sign. Expressive difficulties may be difficult due to factors such as upper limb malformations, dyspraxia, and/or memory for motor movements (Cornelia de Lange Foundation, 2018).

8.2.6 The assessment highlights EG's need for interventions by an occupational therapist. When focusing on the constructs not attained in the Eye and Hand Coordination subscale, the results provide a clear pattern for intervention. It is imperative that a crossover of occupational therapy interventions into a classroom situation occurs. Many of the constructs not achieved fall within the realm of an inability to manipulate objects like using scissors, as well as difficulty reproducing symbolic representations like drawings, rather than manipulating concrete objects like threading beads, which is a precursor to pencil and paper tasks. As gross motor interventions are a relative strength, although not age appropriate, such activities can additionally be addressed in occupational therapy sessions as "fun breaks" after the perceived formal tasks of eye and hand co-ordination if EG has no access to a physiotherapist.Although a relative strength, the Hand and Eye-Co-ordination subscale, when compared to EG's other scores, is far from age appropriate, resulting in an inability to draw and copy basic symbolic representations with accuracy. Occupational therapy intervention at EG's age will provide an opportunity to introduce her to sensory-integrative therapy, as well as assistance with fine motor skill sets. The "autistic-like" rocking can be addressed in these sessions. Swols et al. (2019) state that tactile stimulation during indirection helps children remember and perform maximally. They indicate those activities that stimulate the vestibular system, such as swinging, bouncing, swimming, and horseback riding, are pleasurable to patients with BCdL. These authors report that visuospatial memory and perceptual organization are usually unaffected, but this was not the case with EG. Observations during the assessment indicated that she was able to complete some items, like placing pegs into a pegboard, but the item could not be scored as correct as she did not complete it in the allocated time. Hand and eye-coordination activities form a large part of a pre-school program that EG did not attend resulting in limited exposure to such activities. When 
physical impairments do not limit them, fine motor activities should be stressed in education, especially activities related to activities of daily living.

8.2.7 Myopia, amblyopia, chronic conjunctivitis, photophobia and ptosis are common visual problems in BCdL. Preferential seating close to the blackboard, assigned seating facing away from the windows, adapted but non-glare lighting to highlight the specific work area, and alternating work requiring fine visual functional skills from work requiring less fine visual performance may all be needed in order to reduce fatigue (Cornelia de Lange Foundation, 2018).

8.2.8 Technology provides many options for augmenting communication. However, computers and other high technical devices are not the answer for every child. There are basic skills that must be learned to use this technology effectively. Learners with BCdL need both a basic understanding of the communication process and of the concrete world of objects before they are able to manage the abstract world of symbols (Cornelia De Lange Foundation, 2018).

8.2.9 The Personal-Social-Emotional Subscale constructs not achieved require assistance in the behavioral domain. Behaviors reported in the intake interview including anxiety, rocking, and labile mood, together with constructs not achieved on the above-mentioned subscale, indicate that EG has problems handling frustration, is unable to interpret emotional expressions and does not show concern for others. Her physical appearance is different from typically developing children, which causes varying reactions to her in the community. Psychoeducation about her disorder to staff at her school, her parents, and the community by a psychologist or social worker should assist with acceptance.

8.2.10 The Gross Motor subscale is also a relative strength although the scores indicate that EG is still currently functioning far below her expected age. The manifestations of this delay lie in the realm of balance and movements that rely on complex co-ordination. EG is able to execute activities that require repetitive patterns but is unable to alternate patterns of movement like "hop jump hop". Some of the constructs not achieved on this subscale can be initially addressed in the occupational therapy sessions. Should there be no improvement, a physiotherapy consultation may need to be requested.

8.2.11 Ongoing parental support is necessary to assist with the behaviors experienced at home. Behavioral interventions in the school environment are an integral part of EG's learning process and necessary in an attempt to address behaviors that prevent learning. All professionals involved with her learning must consistently execute interventions introduced.

8.2.12 The co-morbid behaviour that are manifestations of this genetic disorder also require intervention as they affect the learning process, acceptance, attachment and self-esteem. EG's scores on the Conners III indicated hyperactivity, and during the intake interview the parents reported that EG presents with a myriad of behavioural manifestations that include self-injury, severe mood fluctuations and problems with sleeping. EG also engages in autistic-like self-stimulating rocking behaviour. These symptoms are part of the clinical picture. As EG has been diagnosed with $\mathrm{BCdL}$, further investigation into autism based on poor language skills, extreme mood swings and rocking behaviours was not deemed necessary at this stage. 
Other characteristics of her BCdL clinical picture include a diminished ability to relate socially, repetitive and stereotypic behaviorur and infrequent facial expression of emotion. Medical literature reports that $40 \%$ of children diagnosed with BCdL present with hyperactive behaviour, $44 \%$ with daily aggression, and $55 \%$ with sleep disturbance (Chrzanowska, Janniger, \& Wierzba, 2019). These children prefer a structured routine and have difficulty changing it. BCdL syndrome is also associated with a broad range of anxiety disorders, including generalised anxiety disorder, separation anxiety and a diminished ability to relate socially (Swols et al., 2019). These behavioral manifestations are present in EG's daily functioning. These children tend to be hypersensitive and have strong reactions to ordinary stimuli that continue long after the stimulus is no longer present. They also present with irregular patterns of behaviorur in the areas of eating, sleeping and emotional response. The lack of sensitivity to pain or heightened sensitivity to touch suggests some individuals may have neurological impairment and are thus prone to behaviorur problems and often tend to be oppositional.

8.2.13 Psychological interventions for both the child and her parent are integral to support and management. Families may struggle to accept the diagnosis of BCdL. Shock, anger, denial, guilt, and sadness are common early responses. Most families adjust to their new situation, but protracted grief or depression in a family member should be treated. In addition to the initial adjustment, intermittent stresses throughout the life of the child may temporarily destabilize a family.

\section{Challenges}

Although the focus of this article has been on an intervention plan, these ideal plans may not materialize due to various challenges. Four main barriers seem to impede the family's access to appropriate health and medical services. These include location, finance, cultural background and socio-political issues.

8.1 EG's family live in an improvised crime-ridden area populated by gangs. There are no special schooling facilities within a 30-kilometer radius, and only one governmental hospital in the area. These facilities are poorly resourced. Special schooling and health service become difficult to access because of distance. The special schools do not provide transport to this area, making school attendance difficult.

8.2 The parents' financial income places them in a low socioeconomic range. EG's mother is pregnant with a second child and stays at home, while the father earns a minimum wage. The family lives in a two-bedroom brick house and they do not have their own transport.

8.3 The parent's cultural background is complex. Religion plays a pertinent role in their family life and the family frequently look for medical and educational solutions within the church community rather than at resourced institutions. However, emotional support is found within the church and surrounding community. The language spoken in that community is not necessarily the language of tuition in the special school EG may attend.

8.4 Governmental policies govern the resources given to children with special needs. With the introduction of Inclusive Education where special needs children must be accommodated 
in a mainstream school, special schooling is not a priority. Although educational policies advocate education for all children, in reality this does not happen. Special schooling with the necessary assistive devices is expensive and funding is limited, making this type of schooling a scarce resource.

The ideal intervention plan may not be able to be implemented in its current form due to these challenges.

\section{Conclusion}

This article discusses the different challenges that occur when children with delayed development are assessed. The findings highlight that these challenges are not necessarily barriers that need to discourage assessment. Measures, although not normed for these types of children, can be used to obtain sufficient information to design an initial individualised support plan. The Griffiths III was chosen as each of its items assess developmental constructs in five domains at each age level thus allowing the examiner to describe rather than report EG's level of functioning. The process followed serves to guide practitioners working with children with a process that can be followed when faced with the assessment of young children that present with cognitive delays. Each child comes with a different set of challenges that are exacerbated by extraneous variables outside of the family's control and are different for each child. These also need to be factored into the interventions.

The present research consists of a single case study, thus not allowing generalisation of findings and it needs to be acknowledged that multiple case studies may provide further in-depth information. This may not be possible as BCdL is a rare genetic disorder and it would be difficult to access a cohort of such children. Nevertheless the general principles can still be utilised in similar studies of children with developmental delays to assist them with much needed specialised support.

\section{Acknowledgments}

We thank the Association for Research in Infant and Child Development, Charity No.1161043, for the funding the publication fee.

\section{References}

Brassard, M.R., \& Boehm, A.E. (2007). Preschool assessment: Principles and practices. New York. Guildford Press.

Cameron, T. H., \& Kelly, D. P. (1988). Normal Language Skills and Normal Intelligence in a Child with De Lange Syndrome. Journal of Speech and Hearing Research, 53(2), 219-222. http://dx.doi.org/10.1044/jshd.5302.219

Chrzanowska, K. H., Janniger C. K., \& Wierzba, J. (2019). De Lange Syndrome Differential Diagnoses. Retrieved from https://emedicine.medscape.com/article/1116397-differential.html

Conners, C. K. (2008). Conners 3 - Parent and Teacher Surveys, Long Form (Conners 3) Conners 3rd edition manual. Toronto, Ontario, Canada: Multi-Health System. 
Cornelia de Lange Foundation (2018). Characteristics of CdLS. Retrieved from http://cdlsoutreach.org

Green, E., Stroud, L., Bloomfield, S., Cronje, J., Foxcroft, C., Hurter, K. et al. (2016). The Griffiths Scales of Childhood Development, $3^{\text {rd }}$ Edition. Oxford, UK: Hogrefe Ltd.

Griffiths, R. (1954). The abilities of babies. New York, NY: McGraw-Hill.

Janek, K. C., Smith D.F., Kline, J.R., Beneke, Ling Chen, M., Kimball A., \& Ishman S., L. (2016). Improvement of hearing over time in Cornelia de Lange syndrome. Journal of Otorhinolaryngology, 87, 203-207. http://dx.doi.org/10.1016/j.ijporl.2016.06.032

Maloney, E. S., \& Larrivee, L. S. (2017). Limitations of age-equivalent scores in reporting the results of norm-referenced tests. Communication Science and Disorders, 34, 86-93. http://dx.doi.org/10.1044/cicsd_34_F_86

Orsini, A., Pezzuti, L., \& Hulbert, S. (2014). Beyond the floor effect on the Wechsler Intelligence Scale for Children - 4th Ed. (WISC-IV): calculating IQ and Indexes of subjects presenting a floored pattern of results. Journal of Intellectual Disability Research, 59(5), 468-473. http://dx.doi.org/10.1044/cicsd_34_F_86

Sparrow, S. S., \& Cicchetti, D. V. (1989). The Vineland Adaptive Behavior Scales. In C.S. Newmark (Ed), Major psychological assessment instruments, Vol.2 p.199-231, Allyn \& Bacon.

Swols, D.M., Tekin, M., Macdonald, J.T., \& Hussman, J.P (2019) Cornelia de Lange Syndrome: Practice Essentials. Medscape. Retrieved from https://emedicine.medscape.com/article/942792-overview.html

Texas Health and Human Services (2018). Determination of Intellectual Disability:

Best Practice Guidelines. Retrieved from https://hhs.texas.gov/doing-business-hhs/provider-portals/long-term-care-providers/local-intel lectual-developmental-disability-authority-lidda/did-best-practice-guidelines.html

Tseng, M. H., Henderson, A., Chow, S MK., \& Yao, G. (2004) Relationship between motor proficiency, attention, impulse, and activity in children with ADHD. Developmental Medicine and Child Neurology, (46) 6, 381-388. http://dx.doi.org/10.1017/S0012162204000623

Whitaker, S., \& Gordon S. (2012). Floor effects of the WISC IV. International Journal of Developmental Disabilities. $\quad$ 58(2), 111-119. http://dx.doi.org/10.1179/2047387711Y.0000000012

Swols, D.M., Tekin, M., Macdonald, J.T., \& Hussman, J.P (2019) Cornelia de Lange Syndrome: Practice Essentials. Medscape. Retrieved from https://emedicine.medscape.com/article/942792-overview.html 
Texas Health and Human Services (2018). Determination of Intellectual Disability:

Best Practice Guidelines. Retrieved from https://hhs.texas.gov/doing-business-hhs/provider-portals/long-term-care-providers/local-intel lectual-developmental-disability-authority-lidda/did-best-practice-guidelines.html

Tseng, M. H., Henderson, A., Chow, S MK., \& Yao, G. (2004) Relationship between motor proficiency, attention, impulse, and activity in children with ADHD. Developmental Medicine and Child Neurology, (46) 6, 381-388. http://dx.doi.org/10.1017/S0012162204000623

Whitaker, S., \& Gordon S. (2012). Floor effects of the WISC IV. International Journal of Developmental Disabilities. $58(2)$, 111-119. http://dx.doi.org/10.1179/2047387711Y.0000000012

\section{Copyrights}

Copyright for this article is retained by the author(s), with first publication rights granted to the journal.

This is an open-access article distributed under the terms and conditions of the Creative Commons Attribution license (http://creativecommons.org/licenses/by/4.0/) 